\title{
THE EVOLUTION OF EXTRAGALACTIC RADIO SOURCES
}

\author{
A. C. S. READHEAD ${ }^{1}$, T. J. PEARSON ${ }^{1}$, G. B. TAYLOR ${ }^{2}$ AND \\ P. N. WILKINSON ${ }^{3}$
}

\author{
${ }^{1}$ California Institute of Technology \\ ${ }^{2}$ National Radio Astronomy Observatory \\ ${ }^{3}$ University of Manchester
}

A series of VLBI surveys of complete samples of radio sources selected at $5 \mathrm{GHz}$ (Pearson \& Readhead 1988, hereafter PR; Xu et al. 1995 and references therein) has revealed that $\sim 10 \%$ of the objects are "Compact Symmetric Objects" (CSOs), in which high-luminosity radio emission regions are seen on both sides of the center of activity on scales less than one kiloparsec (Phillips \& Mutel 1982; Readhead et al. 1984; Conway et al. 1992; Wilkinson et al. 1994). In order to be sure that an object is a CSO, either the center of activity must be pinpointed (Taylor et al. 1996) or compelling morphological evidence of symmetric structure must be found.

CSOs are unusual amongst high radio luminosity galaxies and quasars because the "working surfaces" of the jets range from a few parsecs to a few hundred parsecs from the center of activity, and thus provide a unique probe of the interstellar medium in the inner kiloparsec. The hot spot pressures of CSOs are $\sim 3 \times 10^{-5}$ dyne $\mathrm{cm}^{-2}$ and the typical distance of PR CSO hot spots from the nucleus are $\sim 50$ pc. We assume that the hot spots are confined by ram pressure with the interstellar medium: the pressure in the hot spot is $\rho_{\mathrm{ext}} v_{a}^{2}$, where $v_{a}$ is the advance speed of the hot spot and $\rho_{\text {ext }}$ is the density of the external medium. The hot spot pressure implies an external density of $10\left(v_{a} / 0.02 c\right)^{-2} \mathrm{~cm}^{-3}$, an age for the PR CSOs of $\sim 10^{4}\left(v_{a} / 0.02 c\right)^{-1} \mathrm{yr}$, and a mass within a $200 \mathrm{pc}$ radius of $\sim 10^{7}\left(v_{a} / 0.02 c\right)^{-2} M_{\odot}$.

Our observations of $2352+495$ (Readhead et al. 1996) enable us to place upper limits on the $\mathrm{HII}$ and $\mathrm{HI}$ density in the external medium of $10^{3} \mathrm{~cm}^{-3}$, which implies an age of less than $10^{5} \mathrm{yr}$. Ages significantly greater than this are ruled out by the implied mass within a 200 pc radius. For example, an age of $10^{6} \mathrm{yr}$ implies a total mass within a $200 \mathrm{pc}$ radius of over $10^{12} M_{\odot}$ in 
the case of $0108+388$, and over $10^{11} M_{\odot}$ in $0710+439$ and $2352+495$. Thus, although we cannot rule out a dense external medium composed mainly of $\mathrm{H}_{2}$ by direct observations, we conclude that, regardless of the state of the external medium ( $\mathrm{HI}, \mathrm{HII}$ or $\mathrm{H}_{2}$ ) the density must be $<10^{3} \mathrm{~cm}^{-3}$, and the age $<10^{5} \mathrm{yr}$. In the case of $2352+495$ the supply time of the energy to the lobes is about $10^{4} \mathrm{yr}$ and there is no evidence of an extended halo into which energetic particles from the lobes are escaping. Thus the lobe energy supply time is likely the age of the lobes. All of the evidence in the PR CSOs is consistent with an age of $10^{4} \mathrm{yr}$ at a size of $50 \mathrm{pc}$. We will, therefore, assume that this is the typical age of the PR CSOs at this size. It is clear that CSOs are much younger than typical Fanaroff-Riley type II objects, and this raises the interesting question of whether CSOs might evolve into CSS doubles (MSOs), and then into large scale FR II objects (Hodges \& Mutel 1987; Fanti et al. 1995). If this unifying evolutionary hypothesis is correct, then it appears that these objects evolve in overall size from $\sim 1$ pc to $\sim 100 \mathrm{kpc}$ with little or no change in expansion speed, in which case the statistics of CSOs, MSOs and large-scale FR II objects show that the luminosity $L$ must decrease approximately according to $L \propto R^{0.3}$, where $R$ is the distance of the jet working surface from the center of activity. This also implies that $\rho_{\text {ext }} \propto R^{-1.3}$ over this range of sizes.

If the above evolutionary scenario is correct, then objects such as Cyg $\mathrm{A}$ would have been about a factor ten more luminous when they were $100 \mathrm{pc}$ in overall size, and the CSO's which comprise $10 \%$ of the high frequency samples we have observed are the precursors of lower luminosity FR II objects. This type of luminosity evolution would have the fortunate consequence that, since objects are more luminous in their early phases, at a given flux density cutoff one is digging deeper into the luminosity function with the smaller sources. This compensates to a considerable degree for the relatively short time that objects spend in this phase, since otherwise there would be very few objects indeed which could be studied in these early phases with high sensitivity.

\section{References}

Conway, J. E., et al. 1992, ApJ, 396, 62

Fanti, C., et al. 1995, A\&A, 302, 317

Hodges, M. W., \& Mutel, R. L. 1987, in Superluminal Radio Sources, Cambridge UP, 168

Pearson, T. J., \& Readhead, A. C. S. 1988, ApJ, 328, 114

Phillips, R. B., \& Mutel, R. L. 1982, A\&A, 106, 21

Readhead, A. C. S., Pearson, T. J., \& Unwin, S. C. 1984, IAU Symp., 110, 131

Readhead, A. C. S., et al. 1996, ApJ, submitted

Taylor, G. B., Readhead, A. C. S., \& Pearson, T. J. 1996, ApJ, submitted

Wilkinson, P. N., et al. 1994, ApJ, 432, L87

Xu, W., et al. 1995, ApJS, 99, 297 